\title{
AN ASYMPTOTIC FORMULA FOR REPRESENTATIONS OF INTEGERS BY INDEFINITE HERMITIAN FORMS
}

\author{
EMILIO A. LAURET
}

(Communicated by Kathrin Bringmann)

\begin{abstract}
We fix a maximal order $\mathcal{O}$ in $\mathbb{F}=\mathbb{R}, \mathbb{C}$ or $\mathbb{H}$, and an $\mathbb{F}$-hermitian form $Q$ of signature $(n, 1)$ with coefficients in $\mathcal{O}$. Let $k \in \mathbb{N}$. By applying a lattice point theorem on an $n$-dimensional $\mathbb{F}$-hyperbolic space, we give an asymptotic formula with an error term, as $t \rightarrow+\infty$, for the number $N_{t}(Q,-k)$ of integral solutions $x \in \mathcal{O}^{n+1}$ of the equation $Q[x]=-k$ satisfying $\left|x_{n+1}\right| \leq t$.
\end{abstract}

\section{INTRODUCTION}

The representation theory by quadratic forms has a long history. It starts with the qualitative problem of determining which integers are represented by a given quadratic form. For example, Fermat, Legendre and Lagrange dealt with the problem of representation as a sum of two, three and four squares respectively. After that, the quantitative problem was considered by Jacobi, Kloosterman and Liouville, among others. For instance, Jacobi proved that the number of ways a positive integer $k$ can be written as a sum of four squares is

$$
8 \sum_{m \mid k, 4 \nmid m} m
$$

by determining the Fourier coefficients of the theta function associated to the form $x_{1}^{2}+\cdots+x_{4}^{2}$.

These examples are for positive definite quadratic forms. For indefinite forms, the literature is much less abundant than for the definite case. In the present work, we study the quantitative problem for quadratic and hermitian forms of signature $(n, 1)$.

Let $\mathbb{F}=\mathbb{R}, \mathbb{C}$ or $\mathbb{H}$, and let $\mathcal{O}$ denote a maximal order in $\mathbb{F}$. Thus $\mathcal{O}$ is the ring of integer numbers $\mathbb{Z}$ in the real case, the ring of integers of an imaginary quadratic extension of $\mathbb{Q}$ in the complex case (e.g., the Gaussian integers $\mathbb{Z}[\sqrt{-1}]$ ), and, for instance, the ring of Hurwitz integers is $\mathbb{F}=\mathbb{H}$, though there are many other choices. We consider in $\mathbb{H}$ (and then by restriction in $\mathbb{C}$ ) the canonical involution $\alpha \mapsto \bar{\alpha}$. An $\mathbb{F}$-hermitian matrix is a square matrix with coefficients in $\mathbb{F}$ that is equal to its own conjugate transpose.

Received by the editors September 29, 2011 and, in revised form, February 22, 2012. 2010 Mathematics Subject Classification. Primary 11D45, 11E39; Secondary 58C40.

Key words and phrases. Representation by hermitian forms, hyperbolic lattice point theorem. This work was supported by CONICET and Secyt-UNC. 
We consider an $\mathbb{F}$-hermitian matrix

$$
Q=\left(\begin{array}{cc}
A & \\
& -a
\end{array}\right)
$$

with $a \in \mathbb{N}$ and $A \in \mathrm{M}_{n}(\mathcal{O})$ a positive definite $\mathbb{F}$-hermitian matrix. We also denote by $Q$ the induced $\mathbb{F}$-hermitian form of signature $(n, 1)$,

$$
Q[x]:=x^{*} Q x=A[\hat{x}]-a\left|x_{n+1}\right|^{2}, \quad x \in \mathbb{F}^{n+1},
$$

where $\hat{x}=\left(x_{1}, \ldots, x_{n}\right)^{t} \in \mathbb{F}^{n}$.

We consider, for $k \in \mathbb{N}$, the solution vectors $x \in \mathcal{O}^{n+1}$ of the equation

$$
Q[x]=-k .
$$

Put

$$
\mathcal{R}(Q,-k)=\left\{x \in \mathcal{O}^{n+1}: Q[x]=-k\right\} .
$$

Since $Q$ is an indefinite form, this set is either empty or infinite. From now on, we will assume it is not empty, i.e., that $-k$ is represented by $Q$. Consequently, in order to study the quantitative behavior, one needs to impose additional restrictions to the integral solutions of (1.2).

A natural condition is to intersect the set in (1.3) with Euclidean balls $\{x \in$ $\left.\mathbb{F}^{n+1}:\|x\| \leq s\right\}$ in $\mathbb{F}^{n+1}$, with $\|x\|^{2}:=A[\hat{x}]+a\left|x_{n+1}\right|^{2}$. Note that this norm is induced by the positive definite $\mathbb{F}$-hermitian matrix $\left(A_{a}\right)$.

A related condition consists of requiring that the solutions of (1.2) satisfy the bound $\left|x_{n+1}\right| \leq t$. We have, for $x \in \mathcal{R}(Q,-k)$, that

$$
\|x\| \leq s \quad \text { if and only if }\left|x_{n+1}\right| \leq \sqrt{\frac{s^{2}+k}{2}} .
$$

In this paper we establish an asymptotic formula, for large $t$, for

$$
N_{t}(Q,-k):=\#\left\{x \in \mathcal{R}(Q,-k):\left|x_{n+1}\right| \leq t\right\},
$$

the number of integral solutions of the equation $Q[x]=-k$ satisfying the additional restriction $\left|x_{n+1}\right| \leq t$.

Our main result, Theorem 5.1, is the asymptotic formula

$$
N_{t}(Q,-k)=\frac{2^{(r-1)(n+1)}}{\left|d_{\mathcal{O}}\right|^{\frac{n+1}{2}}} \frac{a^{\rho} \operatorname{vol}\left(S^{n r-1}\right)}{2 \rho|\operatorname{det} Q|^{\frac{r}{2}}} \frac{\pi^{\frac{r}{2}}}{\Gamma\left(\frac{r}{2}\right)} \delta(Q,-k) t^{2 \rho}+O\left(t^{\tau}\right),
$$

as $t \rightarrow+\infty$, for $\mathbb{F}=\mathbb{R}$ or $\mathbb{C}$. Here $r=\operatorname{dim}_{\mathbb{R}}(\mathbb{F}), \rho=(n+1) r / 2-1, d_{\mathcal{O}}$ is the discriminant of the quotient field of $\mathcal{O}$ and $\delta(Q,-k)$ is the local density of the representation (1.2) (see (4.6)). The number $\tau$ is defined in (3.4). It depends only on the form $Q$ or, more precisely, on the first nonzero eigenvalue of the LaplaceBeltrami operator on $\Gamma_{Q}^{0} \backslash \mathrm{H}_{\mathbb{F}}^{n}$, where $\Gamma_{Q}^{0}$ is a subgroup of the group of unimodular matrices (see 3.7) and $\mathrm{H}_{\mathbb{F}}^{n}$ is the $n$-dimensional $\mathbb{F}$-hyperbolic space. When $\mathbb{F}=\mathbb{R}$ and $n \geq 3$, formula (1.6) holds with $\tau=n-3 / 2$ (note that $2 \rho=n-1$ ).

In the particular case when $\mathbb{F}=\mathbb{R}$ and $Q=I_{n, 1}=\left(\begin{array}{ll}I_{n} & \\ & -1\end{array}\right)$, the main theorem asserts that the number $N_{t}\left(I_{n, 1},-k\right)$ of vectors $x \in \mathbb{Z}^{n+1}$ such that

$$
x_{1}^{2}+\cdots+x_{n}^{2}-x_{n+1}^{2}=-k \quad \text { and } \quad\left|x_{n+1}\right| \leq t
$$

satisfies the following asymptotic estimate, as $t \rightarrow+\infty$ :

$$
N_{t}\left(I_{n, 1},-k\right)=\frac{\operatorname{vol}\left(S^{n-1}\right)}{n-1} \delta\left(I_{n, 1},-k\right) t^{n-1}+O\left(t^{n-3 / 2}\right) .
$$


This formula is due to J. Ratcliffe and S. Tschantz [16]. The present article was inspired by this work. They also considered $k<0$, obtaining the same formula for the leading coefficient without an error term. Although a similar result should hold for $k<0$ in our context, we will not develop this case here since the tools needed are very different.

Concerning the question of counting integral solutions inside Euclidean balls, we define

$$
\widetilde{N}_{s}(Q,-k):=\#\{x \in \mathcal{R}(Q,-k):\|x\| \leq s\} .
$$

By applying formula (1.6) and relation (1.4) (see Remark 5.3), one shows that

$$
\tilde{N}_{s}(Q,-k)=2^{-\rho} C_{Q, k} s^{2 \rho}+O\left(s^{\tau}\right),
$$

where $C_{Q, k}$ denotes the main coefficient in (1.6). Related results for the leading term of the counting function of integral points in quite general algebraic varieties lying in balls of increasing radius as $s \rightarrow+\infty$ have been obtained, for example, in [6] and [2].

In the absence of nonzero exceptional eigenvalues, formulas (1.6) and (1.8) become respectively

$$
\begin{aligned}
& N_{t}(Q,-k)=C_{Q, k} t^{2 \rho}+O\left(t^{2 \rho\left(1-\frac{1}{n+1}\right)+\varepsilon}\right), \\
& \widetilde{N}_{s}(Q,-k)=2^{-\rho} C_{Q, k} s^{2 \rho}+O\left(s^{2 \rho\left(1-\frac{1}{n+1}\right)+\varepsilon}\right),
\end{aligned}
$$

with $\varepsilon=0$ if $\mathbb{F}=\mathbb{R}$ and any $\varepsilon>0$ if $\mathbb{F}=\mathbb{C}$. We recall that $2 \rho=r(n+1)-2$, where $r=\operatorname{dim}_{\mathbb{R}}(\mathbb{F})$.

Our main tool in this paper is the hyperbolic lattice point theorem of P. Lax and R. Phillips [10] in the real case (improved by B. M. Levitan [11]) and of R. Bruggeman, R. Miatello and N. Wallach [4] in the general case $(\mathbb{F}=\mathbb{R}, \mathbb{C}$ and $\mathbb{H})$. For $\mathbb{F}=\mathbb{R}$ we use the best lower bound known for the first eigenvalue of the LaplaceBeltrami operator on $\Gamma_{Q}^{0} \backslash H_{\mathbb{R}}^{n}$ (see Theorem [3.3), which was obtained in [7] and in [5].

After applying the lattice point theorem we obtain (see Proposition 3.6)

$$
N_{t}(Q,-k)=C_{Q, k}^{\prime}\left(\sum_{y \in F}\left|\Gamma_{Q, y}\right|^{-1}\right) t^{2 \rho}+O\left(t^{\tau}\right),
$$

where $C_{Q, k}^{\prime}$ is a constant depending only on $Q$ and $k, F$ is a representative set of $\mathcal{R}(Q,-k)$ under the action of the group of unimodular matrices $\Gamma_{Q}$ (see (2.6) ) and $\Gamma_{Q, y}$ is the stabilizer of $y$ in $\Gamma_{Q}$.

When $\mathbb{F}=\mathbb{R}$ or $\mathbb{C}$, by using the theory of C. L. Siegel [17] for indefinite quadratic forms and its generalization for indefinite complex hermitian forms given in [14, formula (1.9) can be made more explicit, yielding our main formula (1.6). This is carried out by expressing the term $\sum_{y \in F}\left|\Gamma_{Q, y}\right|^{-1}$ as a product of the local density $\delta(Q,-k)$ times a constant which depends only on $Q$ and $k$ (see Corollary 4.3). The derivation of a formula like (1.6) from (1.9) in the quaternionic case would first require developing for indefinite $\mathbb{H}$-hermitian forms the classical theory due to Siegel, as was done by Raghavan (see [14]) for indefinite $\mathbb{C}$-hermitian forms. 
The paper is organized as follows. In Section 2 we introduce the geometric context, relating $N_{t}(Q,-k)$ with the number of elements in an arithmetic subgroup of Iso $\left(\mathrm{H}_{\mathbb{F}}^{n}\right)$ satisfying a geometric condition. In Section 3, we apply the lattice point theorem to count such lattice points. Section 4 uses Siegel's theory to compute the main term of the formula. We conclude with Section 5, which contains the main theorem, together with some examples and remarks.

\section{F-HYPERBOLIC SPACE}

Throughout the paper, given $R$ a ring with identity, we denote by $\mathrm{M}(m, n ; R)$ the set of $m \times n$ matrices with coefficients in $R$, just $\mathrm{M}(m, R)$ when $n=m$, by $\mathrm{GL}(m, \mathbb{F})$ the general linear group and by $\mathrm{SL}(m, \mathbb{F})$ its derived group, the special linear group. For a matrix $C \in \mathrm{M}(m, l ; \mathbb{F})$ we denote by $C^{*}$ its conjugate transpose and write $B[C]=C^{*} B C$, where $B \in \mathrm{M}(m, \mathbb{F})$. Let $R^{m}$ denote the right $R$-module $\mathrm{M}(m, 1 ; R)$. For $\mathbb{F}=\mathbb{R}, \mathbb{C}$ and $\mathbb{H}$, let $\mathrm{P} \mathbb{F}^{n}$ be the $n$-dimensional projective space over $\mathbb{F}$, i.e. $\mathrm{PF}^{n}=\mathbb{F}^{n+1} \backslash\{0\} / \mathbb{F}^{\times}$, where $\mathbb{F}^{\times}$denotes the nonzero elements of $\mathbb{F}$.

We now introduce a model for Riemannian symmetric spaces of real rank one and negative curvature (leaving out the Cayley plane). These are the real, complex and quaternionic hyperbolic spaces. For general references on this subject see [3, II.§10] and [13, §19].

Let $Q$ be the matrix defined as in (1.1). The set

$$
\mathrm{H}_{\mathbb{F}}^{n}(Q)=\left\{[x] \in \mathrm{PF}^{n}: Q[x]<0\right\}
$$

will serve as the set of points for the $Q$-Kleinian model of $n$-dimensional $\mathbb{F}$-hyperbolic geometry. Note that the condition $Q[x]<0$ is well defined on the projective space. The distance function is defined by

$$
\cosh (d([x],[y]))=\frac{|Q(x, y)|}{|Q[x]|^{1 / 2}|Q[y]|^{1 / 2}},
$$

where $Q(x, y)=x^{*} Q y$.

We consider the $\mathbb{F}$-vector space $\mathbb{F}^{n+1}$ endowed with the form $Q(x, y)=x^{*} Q y$ of type $(n, 1)$. Let $x^{\perp}=\left\{u \in \mathbb{F}^{n+1}: Q(x, u)=0\right\}$ be the $Q$-orthogonal complement of $x \in \mathbb{F}^{n+1}$. If $Q[x]<0$, then the restriction of $Q$ to $x^{\perp}$ is positive definite. We identify $x^{\perp}$ with $T_{[x]} \mathrm{H}_{\mathbb{F}}^{n}(Q)$ using the differential of the natural projection $\mathbb{F}^{n+1} \backslash$ $\{0\} \rightarrow \mathrm{H}_{\mathbb{F}}^{n}(Q)$. We consider the symmetric positive definite $\mathbb{R}$-bilinear form on $T_{[x]} \mathrm{H}_{\mathbb{F}}^{n}(Q)$ given by

$$
(u, v)=\frac{\operatorname{Re}(Q(u, v))}{|Q[x]|}, \quad x, y \in x^{\perp} .
$$

In this way $\mathrm{H}_{\mathbb{F}}^{n}(Q)$ is naturally a Riemannian manifold. One can check that the metric associated is (2.2). Moreover, this metric gives constant curvature -1 in the real case and pinched sectional curvature in the interval $[-4,-1]$ if $\mathbb{F}=\mathbb{C}, \mathbb{H}$.

We denote by

$$
\mathrm{U}(Q, \mathbb{F})=\{g \in \mathrm{GL}(n+1, \mathbb{F}): Q[g]=Q\}
$$

the $Q$-unitary group and by $\mathrm{SU}(Q, \mathbb{F})=\mathrm{U}(Q, \mathbb{F}) \cap \mathrm{SL}(n+1, \mathbb{F})$ the special $Q$-unitary group. For $Q=I_{n, 1}$, the classical notation $\mathrm{U}\left(I_{n, 1}, \mathbb{F}\right)=\mathrm{O}(n, 1), \mathrm{U}(n, 1), \operatorname{Sp}(n, 1)$ and $\mathrm{SU}\left(I_{n, 1}, \mathbb{F}\right)=\mathrm{SO}(n, 1), \mathrm{SU}(n, 1), \mathrm{Sp}(n, 1)$ for $\mathbb{F}=\mathbb{R}, \mathbb{C}, \mathbb{H}$ respectively is well 
known. The center of the $Q$-unitary group is given by

$$
Z(\mathrm{U}(Q, \mathbb{F}))= \begin{cases}\left\{ \pm I_{n+1}\right\} & \text { if } \mathbb{F}=\mathbb{R} \\ \left\{z I_{n+1}:|z|=1\right\} \cong S^{1} & \text { if } \mathbb{F}=\mathbb{C} \\ \left\{ \pm I_{n+1}\right\} & \text { if } \mathbb{F}=\mathbb{H} .\end{cases}
$$

The group $\mathrm{U}(Q, \mathbb{F})$ acts transitively on $\mathrm{H}_{\mathbb{F}}^{n}(Q)$ by

$$
[x] \mapsto g \cdot[x]=[g x] .
$$

Indeed, by the distance formula (2.2), its elements act by isometries. Let $\operatorname{Iso}\left(\mathrm{H}_{\mathbb{F}}^{n}(Q)\right)$ (resp. Iso $\left.{ }^{+}\left(\mathrm{H}_{\mathbb{F}}^{n}(Q)\right)\right)$ denote the set of isometries (resp. orientation-preserving isometries) of $\mathrm{H}_{\mathbb{F}}^{n}(Q)$. It is clear that the elements of $Z(\mathrm{U}(Q, \mathbb{F}))$ act as the identity map on $\mathrm{H}_{\mathbb{F}}^{n}(Q)$. Moreover, we have that

$$
\{1\} \longrightarrow Z(\mathrm{U}(Q, \mathbb{F})) \stackrel{\iota}{\longrightarrow} \mathrm{U}(Q, \mathbb{F}) \stackrel{\vartheta}{\longrightarrow} \operatorname{Iso}\left(\mathrm{H}_{\mathbb{F}}^{n}(Q)\right)
$$

is an exact sequence, where $\iota$ denotes the inclusion map and $\vartheta$ is defined by (2.5). Furthermore, the group $\mathrm{PU}(Q, \mathbb{F}):=\mathrm{U}(Q, \mathbb{F}) / Z(\mathrm{U}(Q, \mathbb{F}))$ is, up to finite index, the full isometry group $\operatorname{Iso}\left(\mathrm{H}_{\mathbb{F}}^{n}(Q)\right)$.

Remark 2.1. When $\mathbb{F}=\mathbb{R}$ the group $\mathrm{O}(Q):=\mathrm{U}(Q, \mathbb{R})$ has four connected components. The identity connected component is

$$
\operatorname{PSO}(Q):=\left\{g \in \operatorname{SO}(Q): g_{n+1, n+1}>0\right\},
$$

which is isomorphic to $\mathrm{Iso}^{+}\left(\mathrm{H}_{\mathbb{R}}^{n}(Q)\right)$.

When $\mathbb{F}=\mathbb{C}$, the group $\operatorname{Iso}\left(\mathrm{H}_{\mathbb{C}}^{n}(Q)\right)$ is generated by $\mathrm{PU}(Q, \mathbb{C})$ and the conjugation $\left[x_{1}, \ldots, x_{n+1}\right] \rightarrow\left[\bar{x}_{1}, \ldots, \bar{x}_{n+1}\right]$. If $\mathbb{F}=\mathbb{H}$, then $\operatorname{Iso}\left(\mathrm{H}_{\mathbb{H}}^{n}(Q)\right) \cong \mathrm{PU}(Q, \mathbb{H})$ for $n>1$.

We fix a maximal order $\mathcal{O}$ in $\mathbb{F}$; this means that the subset $\mathcal{O} \subset \mathbb{F}$ satisfies the following conditions:

(i) $\mathcal{O}$ is a lattice in $\mathbb{F}$ (there is an $\mathbb{R}$-basis $v_{1}, \ldots, v_{r}$ of $\mathbb{F}$ such that $\mathcal{O}=$ $\left.\mathbb{Z} v_{1} \oplus \cdots \oplus \mathbb{Z} v_{r}\right)$

(ii) $\mathcal{O}$ is a subring of $\mathbb{F}$ containing 1 ;

(iii) $2 \operatorname{Re}(a) \in \mathbb{Z}$ and $a \bar{a} \in \mathbb{Z}$ for $a \in \mathcal{O}$;

and $\mathcal{O}$ is maximal among all orders (subsets of $\mathbb{F}$ satisfying (i)-(iii)). Here $r:=$ $\operatorname{dim}_{\mathbb{R}}(\mathbb{F})$.

The elements of $\mathcal{O}$ will be called integers. The ring of integers $\mathbb{Z}$ is the only order in $\mathbb{R}$. In $\mathbb{C}$, the maximal orders are the rings of integers of the imaginary quadratic extensions $\mathbb{Q}(\sqrt{-D})(D>0$ squarefree $)$ of $\mathbb{Q}$. More precisely, they are $\mathbb{Z}[\omega] \mathrm{m}$ where $\omega=(1+\sqrt{-D}) / 2$ if $-D \equiv 1(\bmod 4)$ and $\omega=\sqrt{-D}$ otherwise. When $\mathbb{F}=\mathbb{H}$ there are many orders. As a canonical example in this case, the reader may take

$$
\mathcal{O}=\left\{a+b i+c j+d k \in \mathbb{H}: a, b, c, d \in \mathbb{Z} \text { or } a, b, c, d \in \mathbb{Z}+\frac{1}{2}\right\},
$$

the Hurwitz integers.

Let $\Gamma_{Q}$ be the set of unimodular matrices in $\mathrm{U}(Q, \mathbb{F})$, that is,

$$
\Gamma_{Q}=\mathrm{U}(Q, \mathbb{F}) \cap \mathrm{M}(n+1, \mathcal{O}) .
$$

This is a discrete subgroup of $\mathrm{U}(Q, \mathbb{F})$ with finite center. The action of $\Gamma_{Q}$ on $\mathrm{H}_{\mathbb{F}}^{n}(Q)$ is discontinuous, not free, and the quotient $\Gamma_{Q} \backslash \mathrm{H}_{\mathbb{F}}^{n}(Q)$ is of finite volume and not compact. 
On the other hand, the group $\Gamma_{Q}$ acts by left multiplication on the set $\mathcal{R}(Q, k)$ given in (1.3).

Lemma 2.2. The set of $\Gamma_{Q}$-orbits in $\mathcal{R}(Q, k)$ is finite.

Proof. This assertion follows by applying [1, Thm. 6.9].

From now on, we fix $k \in \mathbb{N}$ such that $Q$ represents $-k$; that is, there exists $x \in \mathcal{O}^{n+1}$ satisfying $Q[x]=-k$. Let $F$ be a (finite) set of representatives of the $\Gamma_{Q}$-orbits of $\mathcal{R}(Q,-k)$. Let $\Gamma_{Q, y}$ be the stabilizer of $y$ in $\Gamma_{Q}$, which is finite. We conclude this section by relating the number $N_{t}(Q, k)$ defined in (1.5) with the cardinality of subsets of lattice points in $\Gamma_{Q}$.

Proposition 2.3. For $t>0$, we have

$$
N_{t}(Q,-k)=\sum_{y \in F}\left|\Gamma_{Q, y}\right|^{-1} \#\left\{g \in \Gamma_{Q}: d\left(\left[e_{n+1}\right], g \cdot[y]\right) \leq s\right\},
$$

where $s=\operatorname{arccosh}\left(a^{1 / 2} k^{-1 / 2} t\right)>0$.

Proof. Put

$$
\mathcal{R}_{t}(Q,-k)=\left\{x \in \mathcal{R}(Q,-k):\left|x_{n+1}\right| \leq t\right\} .
$$

The cardinality of this set is $N_{t}(Q,-k)$. Let $x=\left(x_{1}, \ldots, x_{n+1}\right)^{t} \in \mathcal{R}(Q,-k)$. By (2.2),

$$
\cosh \left(d\left(\left[e_{n+1}\right],[x]\right)\right)=a^{1 / 2} k^{-1 / 2}\left|x_{n+1}\right| .
$$

Fix $t>0$; thus $\cosh (s)=a^{1 / 2} k^{-1 / 2} t$. Let $g \in \Gamma_{Q}$ and $y \in F$ be such that $g y=x$; then $g \cdot[y]=[x]$. Note that (2.8) tells us that

$$
\left|x_{n+1}\right| \leq t \quad \text { if and only if } d\left(\left[e_{n+1}\right], g \cdot[y]\right) \leq s .
$$

But the condition on the left ensures that $x \in \mathcal{R}_{t}(Q,-k)$. We conclude that

$$
\mathcal{R}_{t}(Q,-k)=\bigcup_{y \in F}\left\{g y: g \in \Gamma_{Q} \text { and } d\left(\left[e_{n+1}\right], g \cdot[y]\right) \leq s\right\} .
$$

The proposition follows by counting the elements of these sets.

\section{LATTICE POINT THEOREM}

In this section we use lattice point theorems to determine the asymptotic distribution, for $t \rightarrow+\infty$, of the number of elements in the sets

$$
\left\{g \in \Gamma_{Q}: d\left(\left[e_{n+1}\right], g \cdot[y]\right) \leq s\right\} .
$$

Let

$$
G=\mathrm{SU}^{0}(Q, \mathbb{F}),
$$

the identity connected component of $\operatorname{SU}(Q, \mathbb{F})$. We have $G=\operatorname{PSO}(Q)$ for $\mathbb{F}=\mathbb{R}$ (see Remark 2.1). When $\mathbb{F}=\mathbb{C}, \mathbb{H}$ the group $\operatorname{SU}(Q, \mathbb{F})$ is connected. Let $\mathfrak{g}$ denote the Lie algebra $\mathfrak{s u}(Q, \mathbb{F})$ of $G$. We have

$$
\mathfrak{g}=\left\{X \in \mathrm{M}(n+1, \mathbb{F}): X^{*} Q+Q X=0(\text { and } \operatorname{Tr}(X)=0 \text { if } \mathbb{F}=\mathbb{C})\right\} .
$$


Remark 3.1. If $T=\left(\begin{array}{c}{ }^{L} \sqrt{a} \\ { }^{2}\end{array}\right)$, where $L^{*} L=A$, then $I_{n, 1}[T]=T^{*} I_{n, 1} T=Q$. It follows that the map $g \mapsto T g T^{-1}$ gives an isomorphism from $\mathrm{U}(Q, \mathbb{F})$ to $\mathrm{U}(n, 1 ; \mathbb{F}):=$ $\mathrm{U}\left(I_{n, 1}, \mathbb{F}\right)$ and thus also from $G$ to $\mathrm{SU}^{0}(n, 1 ; \mathbb{F})$. The corresponding isomorphism at the Lie algebra level $\mathfrak{s u}(Q, \mathbb{F}) \rightarrow \mathfrak{s u}(n, 1 ; \mathbb{F})$ is given by $X \mapsto T X T^{-1}$. This isomorphism allows us to consider the Cartan involution $\theta$ on $\mathfrak{g}$ by pulling back the standard Cartan involution $X \mapsto-X^{*}$ on $\mathfrak{s u}(n, 1 ; \mathbb{F})$. It is easy to check that $\theta(X)=-\left(T^{*} T\right)^{-1} X^{*}\left(T^{*} T\right)$.

The group $G$ is a connected semisimple Lie group of real rank one and finite center. Let $\theta$ be the Cartan involution given in Remark 3.1, with corresponding Cartan decomposition $\mathfrak{g}=\mathfrak{k} \oplus \mathfrak{p}$. Let $H_{0}$ be the matrix in $\mathfrak{g}$ defined as the pull back from $\mathfrak{s u}(n, 1 ; \mathbb{F})$ of the matrix

$$
\left(\begin{array}{l|l} 
& e_{n} \\
\hline e_{n}^{*} &
\end{array}\right) \text {. }
$$

Let $\mathfrak{a}=\mathbb{R} H_{0}$, a maximal abelian subspace of $\mathfrak{p}$ since $G$ has real rank one. Let $\mathfrak{g}=\mathfrak{k} \oplus \mathfrak{a} \oplus \mathfrak{n}$ and $G=N A K$ be the corresponding Iwasawa decomposition of $\mathfrak{g}$ and $G$, respectively. Here $K$ is a maximal compact subgroup of $G$, with Lie algebra $\mathfrak{k}$. Let $M$ be the centralizer of $A$ in $K$ with Lie algebra $\mathfrak{m}$. Set $\zeta=\operatorname{vol}(K / M)$. Let $2 \rho$ denote the sum of the positive roots of $G$; thus $2 \rho=(n+1) r-2=n-1,2 n$, $4 n+2$ for $\mathbb{F}=\mathbb{R}, \mathbb{C}, \mathbb{H}$ respectively (recall that $r=\operatorname{dim}_{\mathbb{R}}(\mathbb{F})$ ).

Let $B_{K}$ denote the Killing form on $\mathfrak{g}$. We shall work with the inner product $\langle$, on $\mathfrak{g}$ defined by

$$
\langle X, Y\rangle=-\frac{1}{\xi_{\mathbb{F}}} B_{K}(X, \theta Y), \quad \text { where } \xi_{\mathbb{F}}= \begin{cases}2(n-1) & \text { for } \mathbb{F}=\mathbb{R} \\ 4(n+1) & \text { for } \mathbb{F}=\mathbb{C}, \\ 8(n+2) & \text { for } \mathbb{F}=\mathbb{H}\end{cases}
$$

A simple computation shows that $\langle X, Y\rangle=\frac{1}{2} \operatorname{Tr}\left(X^{*} Y\right)$; thus $\left\langle H_{0}, H_{0}\right\rangle=1$. We consider the homogeneous manifold $G / K$, the $G$-invariant Riemannian metric defined by the restriction of $\langle\cdot, \cdot\rangle$ to $\mathfrak{p}$.

One can check that the action of $G$ on $\mathrm{H}_{\mathbb{F}}^{n}(Q)$ given in (2.5) is transitive, the element $\left[e_{n+1}\right]$ lies in $\mathrm{H}_{\mathbb{F}}^{n}(Q)$ and its stabilizer subgroup is $K$. Hence, the map $g \mapsto\left[g e_{n+1}\right]$ from $G$ to $\mathrm{H}_{\mathbb{F}}^{n}(Q)$ gives rise to a $G$-equivariant bijection between the symmetric space $G / K$ and $\mathrm{H}_{\mathbb{F}}^{n}(Q)$. Moreover, it follows by standard arguments that this bijection is already an isometry of Riemannian manifolds. This gives $\mathrm{H}_{\mathbb{F}}^{n}(Q)$ the structure of a Riemannian symmetric space.

Let $\Gamma$ be a non-cocompact lattice in $G$. Set $m_{\Gamma}=|\Gamma \cap Z(G)|$. Let $\operatorname{vol}\left(\Gamma \backslash \mathrm{H}_{\mathbb{F}}^{n}(Q)\right)$ denote the volume on any fundamental domain in $\mathrm{H}_{\mathbb{F}}^{n}(Q)$ relative to $\Gamma_{Q}$. Let $\Delta$ be the Laplace-Beltrami operator on $\Gamma \backslash \mathrm{H}_{\mathbb{F}}^{n}(Q)$. We identify $-\Delta$ with the Casimir operator $C$ of $G$, with respect to the inner product defined in (3.2). We fix a complete orthonormal set $\left\{\varphi_{j}\right\}$ of real valued eigenfunctions of $C$, with eigenvalues $\lambda_{j}$ arranged in increasing order and exceptional eigenvalues $0=\lambda_{0}<\lambda_{1} \leq \cdots \leq$ $\lambda_{N}<\rho^{2}$, which we write as $\lambda_{j}=\rho^{2}-\nu_{j}^{2}$, where $0<\nu_{N} \leq \nu_{N-1} \leq \cdots \leq \nu_{1}<\rho$.

Now we can state the hyperbolic lattice point theorem. It was proved for the real hyperbolic space by Lax and Phillips [10, with an improved error term by Levitan [11, and generalized by Bruggeman, Miatello and Wallach [4 for any symmetric space of real rank one. 
Theorem 3.2. In the notation above, for $[x],[y] \in \mathrm{H}_{\mathbb{F}}^{n}(Q)$, we have that

$$
\begin{aligned}
\#\{g \in \Gamma: d([x], g \cdot[y]) \leq s\}=\frac{2^{1-n} m_{\Gamma} \zeta}{2 \rho \operatorname{vol}\left(\Gamma \backslash \mathrm{H}_{\mathbb{F}}^{n}(Q)\right)} e^{2 \rho s} \\
\quad+2^{1-n} m_{\Gamma} \zeta \sum_{j=1}^{N} \frac{c\left(\nu_{j}\right)}{\nu_{j}+\rho} \varphi_{j}(x) \varphi_{j}(y) e^{\left(\rho+\nu_{j}\right) s}+O\left(e^{\left(2 \rho \frac{n}{n+1}+\varepsilon\right) s}\right)
\end{aligned}
$$

as $s \rightarrow+\infty$, for $\varepsilon=0$ when $\mathbb{F}=\mathbb{R}$ and for any $\varepsilon>0$ otherwise. Here $c(\nu)$ is the Harish-Chandra c-function.

Note that the summation in (3.3) can be restricted to the indices $j$ such that $\rho+\nu_{j}>2 \rho \frac{n}{n+1}$ (in the real case we can replace $>$ by $\geq$ ). Put

$$
\tau= \begin{cases}\rho+\nu_{1} & \text { if } \rho+\nu_{1} \geq 2 \rho \frac{n}{n+1} \quad \text { and } \quad \mathbb{F}=\mathbb{R}, \\ \rho+\nu_{1} & \text { if } \rho+\nu_{1}>2 \rho \frac{n}{n+1} \quad \text { and } \quad \mathbb{F}=\mathbb{C}, \mathbb{H}, \\ 2 \rho \frac{n}{n+1}+\varepsilon & \text { otherwise, }\end{cases}
$$

where $\varepsilon$ is zero if $\mathbb{F}=\mathbb{R}$ or any positive value if $\mathbb{F}=\mathbb{C}, \mathbb{H}$. The last case includes the case when there are no exceptional eigenvalues. With this notation we can rewrite (3.3) as

$$
\#\{g \in \Gamma: d([x], g \cdot[y]) \leq s\}=\frac{2^{1-n} m_{\Gamma} \zeta}{2 \rho \operatorname{vol}\left(\Gamma \backslash \mathrm{H}_{\mathbb{F}}^{n}(Q)\right)} e^{2 \rho s}+O\left(e^{\tau s}\right) .
$$

We see that in (3.5) the error term depends on the first nonzero eigenvalue of the Laplace-Beltrami operator on $\Gamma \backslash \mathrm{H}_{\mathbb{F}}^{n}(Q)$. The following theorem was proved in [7. Thm. A] (see also [5]). The notation $\operatorname{PSO}(Q)$ was introduced in Remark 2.1.

Theorem 3.3. Let $n \geq 3$ and let $Q_{0}$ be a quadratic form with rational coefficients such that $Q_{0}$ is of signature $(n, 1)$ and isotropic over $\mathbb{Q}$. For any congruence subgroup $\Gamma<\operatorname{PSO}\left(Q_{0}\right)$, the first nonzero eigenvalue $\lambda_{1}$ for the Laplace-Beltrami operator on $\Gamma \backslash \mathrm{H}_{\mathbb{R}}^{n}(Q)$ satisfies $\lambda_{1} \geq(2 n-3) / 4$.

Bounds of this kind are not known in the complex and quaternionic case (see [12, Cor. 1.4] for a related result in the complex case).

Remark 3.4. Under the assumptions of Theorem 3.3, the value of $\nu_{1}$ satisfies

$$
\nu_{1} \leq \sqrt{\left(\frac{n-1}{2}\right)^{2}-\frac{2 n-3}{4}}=\frac{n-2}{2} .
$$

Moreover, in the worst possible case $\nu_{1}=\frac{n-2}{2}$, one has $\rho+\nu_{1} \geq 2 \rho \frac{n}{n+1}$ for all $n \geq 3$, which implies that (3.5) holds for $\tau=\frac{n-1}{2}+\frac{n-2}{2}=n-\frac{3}{2}$ since $\mathbb{F}=\mathbb{R}$.

Before applying Theorem 3.2 to our problem, we give the value of $\zeta=\operatorname{vol}(K / M)$.

Lemma 3.5. Under the notation above, one has $\zeta=\operatorname{vol}\left(S^{n r-1}\right)$.

Proof. It is sufficient to prove the lemma for $Q=I_{n, 1}$ since the isomorphism between $G$ and $\mathrm{SU}^{0}(n, 1 ; \mathbb{F})$ given in Remark 3.1 preserves the Killing form and the inner product (3.2).

Set $S=\{H \in \mathfrak{p}:\langle H, H\rangle=1\}$ with the Riemannian metric given by the real inner product $\langle\cdot, \cdot\rangle$ restricted to $\{X \in \mathfrak{p}:\langle X, H\rangle=0\} \cong T_{H} S$. The adjoint representation restricted to $K$ leaves the set $S$ invariant; this action is transitive 
and the stabilizer subgroup of $H_{0} \in S$ is $M$. Then, we have a $K$-equivariant bijection from the manifold $K / M$ to $S$, which is already an isometry, considering the Riemannian metric on $K / M$ given by (3.2) restricted to $\mathfrak{k} \cap \mathfrak{m}^{\perp}$.

It remains to prove that the Riemannian manifold $S$ is isometric to the $(n r-1)$ dimensional sphere in $\mathbb{R}^{n r}$. It can be checked that

$$
\mathfrak{p}=\left\{X_{v}=\left(\begin{array}{c|c} 
& v \\
\hline v^{*} &
\end{array}\right): v \in \mathbb{F}^{n}\right\} .
$$

Then $\left\langle X_{v}, X_{w}\right\rangle=\frac{1}{2} \operatorname{Tr}\left(X_{v}^{*} X_{w}\right)=\operatorname{Re}\left(\sum_{l=1}^{n} \bar{v}_{l} w_{l}\right)$. Identifying $\mathfrak{p}$ with $\mathbb{F}^{n} \cong \mathbb{R}^{r n}$ in the obvious way, the inner product $\langle\cdot, \cdot\rangle$ on $\mathfrak{p}$ coincides with the canonical inner product on the (real) vector space $\mathbb{F}^{n}$ of dimension $n r$. Hence $S$ is the $(n r-1)$ dimensional sphere of radius one in the (standard) Euclidean space $\mathbb{R}^{n r}$.

For a more detailed description of the realizations of the symmetric spaces $G / K$ and $K / M$, we refer to $[9, \S 10, \mathrm{Ch}$. XI].

Let

$$
\Gamma_{Q}^{0}=G \cap \Gamma_{Q}=\operatorname{SU}^{0}(Q, \mathbb{F}) \cap \mathrm{M}(n+1, \mathcal{O}) .
$$

This is a finite index subgroup of $\Gamma_{Q}$ (see (2.6) $)$. Let us denote by $w$ the number of units in $\mathcal{O}$ when $\mathbb{F}=\mathbb{R}$ or $\mathbb{C}$. It is clear that

$$
\kappa:=\left[\Gamma_{Q}: \Gamma_{Q}^{0}\right]= \begin{cases}4 & \text { if } \mathbb{F}=\mathbb{R} \\ w & \text { if } \mathbb{F}=\mathbb{C}, \\ 1 & \text { if } \mathbb{F}=\mathbb{H} .\end{cases}
$$

Let $\left\{g_{1}, \ldots, g_{\kappa}\right\}$ be a set of representatives of the $\Gamma_{Q^{-c o c l a s s e s}}^{0} \Gamma_{Q}$. For example, $\left\{\left(\begin{array}{lll}I_{n-1} & & \\ & \pm 1 & \\ & & \pm 1\end{array}\right)\right\}$ if $\mathbb{F}=\mathbb{R},\left\{\left(\begin{array}{ll}I_{n} & \alpha\end{array}\right): \alpha \in \mathcal{O}^{\times}\right\}$if $\mathbb{F}=\mathbb{C}$ and $\left\{I_{n+1}\right\}$ if $\mathbb{F}=\mathbb{H}$.

Now we can apply the lattice point theorem to our problem. For each $1 \leq j \leq \kappa$, Theorem 3.2 implies that

$$
\#\left\{g \in \Gamma_{Q}^{0}: d\left(\left[e_{n+1}\right], g \cdot\left(g_{j} \cdot[y]\right)\right) \leq s\right\}=\frac{2^{1-n} m_{\Gamma_{Q}^{0}} \zeta}{2 \rho \operatorname{vol}\left(\Gamma_{Q}^{0} \backslash \mathrm{H}_{\mathbb{F}}^{n}(Q)\right)} e^{2 \rho s}+O\left(e^{s \tau}\right) .
$$

A trivial verification shows that $\operatorname{vol}\left(\Gamma_{Q}^{0} \backslash \mathrm{H}_{\mathbb{F}}^{n}(Q)\right)=\xi_{\mathbb{F}} \operatorname{vol}\left(\Gamma_{Q} \backslash \mathrm{H}_{\mathbb{F}}^{n}(Q)\right)$, with $\xi_{\mathbb{R}}=2$, $\xi_{\mathbb{C}}=m_{\Gamma_{Q}^{0}}$ and $\xi_{\mathbb{H}}=1$. Furthermore, Lemma 3.5 gives $\zeta=\operatorname{vol}\left(S^{n r-1}\right)$. These considerations imply, by adding formula (3.8) over $j$, that

$$
\#\left\{g \in \Gamma_{Q}: d\left(\left[e_{n+1}\right], g \cdot[y]\right) \leq s\right\}=\widetilde{w} \frac{2^{1-n} \operatorname{vol}\left(S^{n r-1}\right)}{2 \rho \operatorname{vol}\left(\Gamma_{Q} \backslash \mathrm{H}_{\mathbb{F}}^{n}(Q)\right)} e^{2 \rho s}+O\left(e^{s \tau}\right),
$$

where $\widetilde{w}=w$ if $\mathbb{F}=\mathbb{R}, \mathbb{C}$ and $\widetilde{w}=2$ if $\mathbb{F}=\mathbb{H}$. Applying this formula to (2.7), we obtain that

$$
N_{t}(Q,-k)=\widetilde{w} \frac{2^{1-n} \operatorname{vol}\left(S^{n r-1}\right)}{2 \rho \operatorname{vol}\left(\Gamma_{Q} \backslash H_{\mathbb{F}}^{n}(Q)\right)}\left(\sum_{y \in F}\left|\Gamma_{Q, y}\right|^{-1}\right) e^{2 \rho s}+O\left(e^{s \tau}\right) .
$$

Recall that $\cosh (s)=a^{1 / 2} k^{-1 / 2} t$. Notice that we can replace the error term in (3.10) by $O\left(t^{\tau}\right)$ since $e^{s} \sim 2 \cosh (s)=2 a^{1 / 2} k^{-1 / 2} t$ as $s \rightarrow+\infty$, and furthermore $e^{2 \rho s}=2^{2 \rho} a^{\rho} k^{-\rho} t^{2 \rho}+O\left(t^{\tau}\right)$. 
Collecting all the information in this section, we have obtained the following formula.

Proposition 3.6. The number $N_{t}(Q,-k)$ satisfies the asymptotic estimate

$$
N_{t}(Q,-k)=\widetilde{w} \frac{2^{2 \rho-(n-1)} a^{\rho} \operatorname{vol}\left(S^{n r-1}\right)}{2 \rho k^{\rho} \operatorname{vol}\left(\Gamma_{Q} \backslash \mathrm{H}_{\mathbb{F}}^{n}(Q)\right)}\left(\sum_{y \in F}\left|\Gamma_{Q, y}\right|^{-1}\right) t^{2 \rho}+O\left(t^{\tau}\right),
$$

as $t \rightarrow+\infty$, where $\tau$ is as in (3.4) and $\widetilde{w}=w$ if $\mathbb{F}=\mathbb{R}, \mathbb{C}$ and $\widetilde{w}=2$ if $\mathbb{F}=\mathbb{H}$. Moreover, when $\mathbb{F}=\mathbb{R}$ and $n>2$, (3.11) holds with $\tau=n-3 / 2$.

The last assertion follows from Remark 3.4

\section{THE MASS OF THE REPRESENTATION}

The object of this section is to obtain a formula for the term $\sum_{y \in F}\left|\Gamma_{Q, y}\right|^{-1}$ by using Siegel's theory on quadratic forms and its generalization to complex hermitian forms given by Raghavan. Our main references are [18, 15] and 14. From now on we make the assumption $\mathbb{F}=\mathbb{R}$ or $\mathbb{C}$. We denote by $d_{\mathcal{O}}$ the discriminant of the quotient field of $\mathcal{O}$. The following remark introduces a canonical volume element in algebraic varieties that will be useful (see [18, $\S 5, \mathrm{Ch}$. IV] for more details).

Remark 4.1. Let $x_{1}, \ldots, x_{m}$ denote the coordinates of $\mathbb{R}^{m}$. Let $y_{j}=f_{j}\left(x_{1}, \ldots, x_{m}\right)$ $(1 \leq j \leq n)$ be $n$ smooth functions with $n \leq m$. Let $a_{1}, \ldots, a_{n} \in \mathbb{R}$ such that the surface $\Omega=\left\{x \in \mathbb{R}^{m}: y_{j}(x)=a_{j}\right.$, for $\left.1 \leq j \leq n\right\}$ is nonsingular; that is, the matrix with entries $\frac{\partial f_{i}}{\partial x_{j}}$ has maximum rank $n$ at every point of $\Omega$. Choose $m-n$ differentiable functions $y_{n+1}, \ldots, y_{m}$ of $\mathbb{R}^{m}$ so that the Jacobian $J=\operatorname{det}\left(\frac{\partial y_{i}}{\partial x_{j}}\right)$ is nonzero at every point of $\Omega$. Then

$$
d \omega=|J|^{-1} d y_{n+1} \ldots d y_{m}
$$

gives a volume element on $\Omega$ independently of the choice of $y_{n+1}, \ldots, y_{m}$. Siegel denoted this volume element by $\frac{\{d x\}}{\{d y\}}$.

We pick $v \in \mathbb{F}^{n}$ and $R \in \mathrm{M}(n, \mathbb{F})$ such that the matrix

$$
W=\left(\begin{array}{cc}
-k & v \\
v^{t} & R
\end{array}\right)
$$

is $\mathbb{F}$-hermitian of signature $(n, 1)$. For $y \in \mathbb{F}^{n+1}$ such that $Q[y]=-k$, let $\mathrm{U}(Q, \mathbb{F})_{y}$ denote the set of elements $U \in \mathrm{U}(Q, \mathbb{F})$ such that $U y=y$. Note that if $y \in \mathcal{O}^{n+1}$, the stabilizer of $y$ in $\Gamma_{Q}$ is $\Gamma_{Q, y}=\mathrm{U}(Q, \mathbb{F})_{y} \cap \mathrm{GL}(n+1, \mathcal{O})$. Consider the varieties

$$
\begin{aligned}
\Omega(Q, W) & =\{X \in \mathrm{M}(n+1, \mathbb{F}): Q[X]=W\}, \\
\Omega(Q, W ; y) & =\{Y \in \mathrm{M}(n+1, n ; \mathbb{F}): Q[(y \mid Y)]=W\} .
\end{aligned}
$$

The groups $\mathrm{U}(Q, \mathbb{F})$ and $\mathrm{U}(Q, \mathbb{F})_{y}$ act by left multiplication on $\Omega(Q, W)$ and on $\Omega(Q, W ; y)$ respectively. On these varieties we fix the volume elements

$$
d \omega=\left|\frac{\operatorname{det}(W)}{\operatorname{det}(Q)}\right|^{\frac{2-r}{2}} \frac{\{d X\}}{\{d W\}} \quad \text { and } \quad d \omega^{*}=\left|\frac{\operatorname{det}(W)}{\operatorname{det}(Q)}\right|^{\frac{2-r}{2}} \frac{\{d X\}}{\{d v\}\{d R\}},
$$

respectively, where $\frac{\{d X\}}{\{d W\}}$ and $\frac{\{d X\}}{\{d v\}\{d R\}}$ are the volume elements given in Remark 4.1. In the complex case, we write $X=X^{(1)}+i X^{(2)}$ and $W=W^{(1)}+i W^{(2)}$ with $X^{(1)}$, $X^{(2)}, W^{(1)}$ and $W^{(2)}$ real matrices. Thus, the volume element $\frac{\{d X\}}{\{d W\}}$ is defined by 
considering the algebraic equations $\operatorname{Re}\left(Q\left[X^{(1)}+i X^{(2)}\right]\right)=W^{(1)}$ and $\operatorname{Im}\left(Q\left[X^{(1)}+\right.\right.$ $\left.\left.i X^{(2)}\right]\right)=W^{(2)}$. The factor $|\operatorname{det}(W) / \operatorname{det}(Q)|^{\frac{2-r}{2}}$ is included so that $d \omega$ does not depend on $W$. Similar considerations apply for $\frac{\{d X\}}{\{d v\}\{d R\}}$ and $d \omega^{*}$.

Siegel (and [15] for the hermitian case) defines the measure of the representation of $-k \in \mathbb{Z}$ by $Q$ as

$$
\mu(Q,-k)=\sum_{y \in F} \mu(y, Q) / \mu(Q) .
$$

Here $\mu(Q)$ denotes the measure of the unit group $\Gamma_{Q}$ given by $\left(r^{2} /\left|d_{\mathcal{O}}\right|\right)^{(n+1)(n+2) / 4}$ times the volume of any fundamental domain for the action of $\Gamma_{Q}$ on $\Omega(Q, W)$, and, similarly, $\mu(y, Q)$ is the measure of the representation $y$ given by $\left(r^{2} /\left|d_{\mathcal{O}}\right|\right)^{n(n+1) / 4}$ times the volume of any fundamental domain for the action of $\Gamma_{Q, y}$ on $\Omega(Q, W ; y)$.

We will recover from the right hand side of (4.2) the term $\sum_{y \in F}\left|\Gamma_{Q, y}\right|^{-1}$ and then, by applying Siegel's main theorem, we will obtain an explicit formula for this term. By [18, Thm. 7, Ch. IV] and [14, (93)] (or [15, (70)]) we have that

$$
w \mu(Q)=\left(r^{2} /\left|d_{\mathcal{O}}\right|\right)^{\frac{(n+1)(n+2)}{4}} \frac{\operatorname{vol}\left(\Gamma_{Q} \backslash \mathrm{H}_{\mathbb{F}}^{n}(Q)\right)}{r^{n+1}|\operatorname{det} Q|^{\frac{r}{2} n+1}} \frac{\pi^{\frac{r}{2}}}{\Gamma\left(\frac{r}{2}\right)} \prod_{j=1}^{n} \frac{\pi^{\frac{r}{2} j}}{\Gamma\left(\frac{r}{2} j\right)},
$$

where $w=\# \mathcal{O}^{\times}$.

Let $\widetilde{\mathcal{F}}(y)$ be a fundamental domain of the action of $\Gamma_{Q, y}$ on $\Omega(Q, W ; y)$. By definition $\mu(y, Q)=|\operatorname{det} W|^{(2-r) / 2}|\operatorname{det} Q|^{-(2-r) / 2}\left(r^{2} /\left|d_{\mathcal{O}}\right|\right)^{n(n+1) / 4} \int_{\widetilde{\mathcal{F}}(y)} d \omega^{*}$, but the measure $d \omega^{*}$ is invariant by $\Gamma_{Q, y}$. Then

$$
\mu(y, Q)=\frac{1}{\left|\Gamma_{Q, y}\right|}\left|\frac{\operatorname{det} W}{\operatorname{det} Q}\right|^{(2-r) / 2}\left(r^{2} /\left|d_{\mathcal{O}}\right|\right)^{\frac{n(n+1)}{4}} \int_{\Omega(Q, W ; y)} d \omega^{*} .
$$

Using [18, Thm. 6, Ch. IV] and [15, Lemma 9], we have that

$$
\mu(y, Q)=\left|\Gamma_{Q, y}\right|^{-1}\left(r^{2} /\left|d_{\mathcal{O}}\right|\right)^{\frac{n(n+1)}{4}} \frac{k^{1-\frac{r}{2}(n+1)}}{|\operatorname{det} Q|^{\frac{r}{2}(n-1)+1}} \prod_{j=1}^{n} \frac{\pi^{\frac{r}{2} j}}{\Gamma\left(\frac{r}{2} j\right)} .
$$

Finally, (4.3) and (4.4) imply that

$$
\mu(Q,-k)=w\left|d_{\mathcal{O}}\right|^{\frac{n+1}{2}} \frac{k^{1-\frac{r}{2}(n+1)}|\operatorname{det} Q|^{\frac{r}{2}}}{\operatorname{vol}\left(\Gamma_{Q} \backslash \mathrm{H}_{\mathbb{F}}^{n}(Q)\right)} \frac{\Gamma\left(\frac{r}{2}\right)}{\pi^{\frac{r}{2}}} \sum_{y \in F}\left|\Gamma_{Q, y}\right|^{-1} .
$$

Now, we will recall Siegel's main theorem for indefinite quadratic and hermitian forms (see [17, Thm. 1] and [14, Thm. 7]). For every rational prime $p$, the $p$-adic density of representation of $-k$ by $Q$ is

$$
\delta_{p}(Q,-k)=\lim _{j \rightarrow \infty} p^{-j(r(n+1)-1)} \#\left\{x \in\left(\mathcal{O} / p^{j} \mathcal{O}\right)^{n+1}: Q[x] \equiv-k \bmod p^{j}\right\} .
$$

Define $\delta(Q,-k)=\prod_{p} \delta_{p}(Q,-k)$, the local density, where the product is over all prime numbers.

Theorem 4.2 (Siegel's mass formula). Let $Q$ be an $\mathbb{F}$-hermitian form as in (1.1) with $n \geq 2$ and let $k$ be a positive integer. Then

$$
\mu(Q,-k)=\delta(Q,-k) .
$$


See [17, Thm. 1] for the real case and [14, Thm. 7] for the complex case.

By combining equation (4.5) and Theorem 4.2. we obtain an expression for the term $\sum_{y \in F}\left|\Gamma_{Q, y}\right|^{-1}$, the main goal of this section.

Corollary 4.3. We have that

$$
\sum_{y \in F}\left|\Gamma_{Q, y}\right|^{-1}=w^{-1}\left|d_{\mathcal{O}}\right|^{-\frac{n+1}{2}} \frac{k^{\frac{r}{2}(n+1)-1}}{|\operatorname{det} Q|^{\frac{r}{2}}} \operatorname{vol}\left(\Gamma_{Q} \backslash \mathrm{H}_{\mathbb{F}}^{n}(Q)\right) \frac{\pi^{\frac{r}{2}}}{\Gamma\left(\frac{r}{2}\right)} \delta(Q,-k) .
$$

Note that $\pi^{r / 2} / \Gamma(r / 2)=1, \pi, \pi^{2}$ for $\mathbb{F}=\mathbb{R}, \mathbb{C}$ and $\mathbb{H}$ respectively.

\section{MAIN THEOREM}

We can now state the main result in this paper, which follows by combining Proposition 3.6 and Corollary 4.3. The last assertion is a consequence of Remark 3.4. We first recall some terminology: $r=\operatorname{dim}_{\mathbb{R}}(\mathbb{F}), \rho=(n+1) r / 2-1$ and $\mathcal{O}$ is a maximal order in $\mathbb{F}$ with discriminant $d_{\mathcal{O}}$.

Theorem 5.1. Let $Q$ be an $\mathbb{F}$-hermitian matrix as in (1.1), with $n \geq 2$ and $\mathbb{F}=\mathbb{R}$ or $\mathbb{C}$. We fix $k \in \mathbb{N}$ such that $-k$ is represented by $Q$. Then, the number $N_{t}(Q,-k)$ of elements $x \in \mathcal{O}^{n+1}$ such that $Q[x]=-k$ and $\left|x_{n+1}\right| \leq t$ satisfies the following asymptotic estimate as $t \rightarrow+\infty$ :

$$
N_{t}(Q,-k)=\frac{2^{(r-1)(n+1)}}{\left|d_{\mathcal{O}}\right|^{\frac{n+1}{2}}} \frac{a^{\rho} \operatorname{vol}\left(S^{n r-1}\right)}{2 \rho|\operatorname{det} Q|^{\frac{r}{2}}} \frac{\pi^{\frac{r}{2}}}{\Gamma\left(\frac{r}{2}\right)} \delta(Q,-k) t^{2 \rho}+O\left(t^{\tau}\right),
$$

where $\tau$ is as in (3.4). Moreover, when $\mathbb{F}=\mathbb{R}$ and $n>2$, formula (3.11) holds for $\tau=n-3 / 2$.

Remark 5.2. In order to get an explicit value of the main term in (5.1) for a fixed $\mathbb{F}$-hermitian form $Q$, one needs to determine $\delta(Q,-k)$. When $\mathbb{F}=\mathbb{R}$, T. Yang [19] computed this local density for any quadratic form $Q$. For $\mathbb{F}=\mathbb{C}$ see $[8$.

Remark 5.3. We consider the real norm $\|\cdot\|$ induced by the $\mathbb{F}$-hermitian form $\left({ }^{A}{ }_{a}\right)$, i.e. $\|x\|^{2}=A[\hat{x}]+a\left|x_{n+1}\right|^{2}$, where $\hat{x}=\left(x_{1}, \ldots, x_{n}\right)^{t}$. We claim that Theorem 5.1 provides an asymptotic formula with error term for $\tilde{N}_{s}(Q,-k)$ (see (1.7)), as $s \rightarrow$ $+\infty$, of the number of solutions lying in the Euclidean ball of radius $s$. Indeed, it is clear that

$$
\left.\begin{array}{rl}
Q[x] & =A[\hat{x}]-a\left|x_{n+1}\right|^{2}=-k \\
\|x\|^{2} & =A[\hat{x}]+a\left|x_{n+1}\right|^{2} \leq s^{2}
\end{array}\right\} \quad \text { imply } \quad\left|x_{n+1}\right| \leq \sqrt{\frac{s^{2}+k}{2}}=: t_{s} .
$$

Hence, by Theorem 5.1, we have

$$
\tilde{N}_{s}(Q,-k)=N_{t_{s}}(Q,-k)=C_{Q, k} t_{s}^{2 \rho}+O\left(t_{s}^{\tau}\right),
$$

where $C_{Q, k}$ denotes the main coefficient of (5.1). By Taylor's expansion at $s=\infty$, $t_{s}=s / \sqrt{2}+O\left(s^{-1}\right)$; thus we can replace $O\left(t_{s}^{\tau}\right)$ by $O\left(s^{\tau}\right)$ in (5.2). Moreover, $t_{s}^{2 \rho}=s^{2 \rho} / 2^{\rho}+O\left(s^{2 \rho-2}\right)$. But $\tau \geq 2 \rho n /(n+1)+\varepsilon$ by (3.4), and so it follows immediately that $\tau-(2 \rho-2)>0$ for $\mathbb{F}=\mathbb{R}$ and $\mathbb{C}$. Therefore, (5.2) reduces to

$$
\widetilde{N}_{s}(Q,-k)=2^{-\rho} C_{Q, k} s^{2 \rho}+O\left(s^{\tau}\right) .
$$


Example 5.4. The cases $\mathbb{F}=\mathbb{R}$ and $Q=I_{n, 1}=\left({ }^{I_{n}}{ }_{-1}\right)$ were considered by J. Ratcliffe and S. Tschantz [16]. We have $r=1,\left|d_{\mathcal{O}}\right|=1, a=1,\left|\operatorname{det}\left(I_{n, 1}\right)\right|=1$ and $\rho=(n-1) / 2$. Theorem 5.1 now yields

$$
N_{t}\left(I_{n, 1},-k\right)=\frac{\operatorname{vol}\left(S^{n-1}\right)}{n-1} \delta\left(I_{n, 1},-k\right) t^{n-1}+O\left(t^{n-3 / 2}\right) .
$$

In [16, Thm. 12] there is an explicit formula for the local density $\delta\left(I_{n, 1},-k\right)$.

Example 5.5. We conclude the article by considering the case of the Lorentzian hermitian form over the Gaussian integers, i.e. $Q=I_{n, 1}$ for $\mathbb{F}=\mathbb{C}$ and $\mathcal{O}=\mathbb{Z}[\sqrt{-1}]$. We have $r=2, \rho=n, d_{\mathcal{O}}=-4, a=1$ and $\left|\operatorname{det}\left(I_{n, 1}\right)\right|=1$. Furthermore, $\operatorname{vol}\left(S^{2 n-1}\right)=2 \pi^{n} /(n-1)$ !. Theorem 5.1 now implies that

$$
N_{t}\left(I_{n, 1},-k\right)=\frac{\pi^{n+1}}{n !} \delta\left(I_{n, 1},-k\right) t^{2 n}+O\left(t^{\tau}\right) .
$$

The local density $\delta\left(I_{n, 1},-k\right)$ is explicitly computable. For example, when $n=2$ and $k=1$ we have $\delta\left(I_{2,1},-1\right)=2^{3} 3 \pi^{-3}$, and consequently $N_{t}\left(I_{2,1},-1\right)=12 t^{4}+O\left(t^{\tau}\right)$. In a future paper we will compute this term for several examples and test our formula with experimental computations.

\section{ACKNOWLEDGMENTS}

This is part of the author's Ph.D. thesis, written under the supervision of Professor Roberto Miatello at the Universidad Nacional de Córdoba, Argentina. The author sincerely thanks Roberto for suggesting the problem and for many helpful discussions concerning the material in this paper. The author also wishes to express his thanks to Wai Kiu Chan, Rainer Schulze-Pillot and Takao Watanabe for several helpful comments and to Jorge Lauret and the referee for reading the draft and making helpful suggestions.

\section{REFERENCES}

[1] Armand Borel and Harish-Chandra, Arithmetic subgroups of algebraic groups, Ann. of Math. (2) 75 (1962), 485-535. MR0147566 (26 \#5081)

[2] Mikhail Borovoi and Zeév Rudnick, Hardy-Littlewood varieties and semisimple groups, Invent. Math. 119 (1995), no. 1, 37-66, DOI 10.1007/BF01245174. MR1309971 (96b:11084)

[3] Martin R. Bridson and André Haefliger, Metric spaces of non-positive curvature, Grundlehren der Mathematischen Wissenschaften [Fundamental Principles of Mathematical Sciences], vol. 319, Springer-Verlag, Berlin, 1999. MR1744486 (2000k:53038)

[4] R. W. Bruggeman, R. J. Miatello, and N. R. Wallach, Resolvent and lattice points on symmetric spaces of strictly negative curvature, Math. Ann. 315 (1999), no. 4, 617-639, DOI 10.1007/s002080050331. MR 1731464 (2001f:11170)

[5] J. Cogdell, J.-S. Li, I. Piatetski-Shapiro, and P. Sarnak, Poincaré series for SO(n,1), Acta Math. 167 (1991), no. 3-4, 229-285, DOI 10.1007/BF02392451. MR1120604 (93h:11050)

[6] W. Duke, Z. Rudnick, and P. Sarnak, Density of integer points on affine homogeneous varieties, Duke Math. J. 71 (1993), no. 1, 143-179, DOI 10.1215/S0012-7094-93-07107-4. MR.1230289(94k:11072)

[7] J. Elstrodt, F. Grunewald, and J. Mennicke, Kloosterman sums for Clifford algebras and a lower bound for the positive eigenvalues of the Laplacian for congruence subgroups acting on hyperbolic spaces, Invent. Math. 101 (1990), no. 3, 641-685, DOI 10.1007/BF01231519. MR.1062799 (91j:11038)

[8] Yumiko Hironaka, Spherical functions and local densities on Hermitian forms, J. Math. Soc. Japan 51 (1999), no. 3, 553-581, DOI 10.2969/jmsj/05130553. MR.1691493(2000c:11064) 
[9] Shoshichi Kobayashi and Katsumi Nomizu, Foundations of differential geometry. Vol. II, Interscience Tracts in Pure and Applied Mathematics, No. 15, Vol. II, Interscience Publishers John Wiley \& Sons, Inc., New York-London-Sydney, 1969. MR0238225 (38 \#6501)

[10] Peter D. Lax and Ralph S. Phillips, The asymptotic distribution of lattice points in Euclidean and non-Euclidean spaces, J. Funct. Anal. 46 (1982), no. 3, 280-350, DOI 10.1016/00221236(82)90050-7. MR661875 (83j:10057)

[11] B. M. Levitan, Asymptotic formulas for the number of lattice points in Euclidean and Lobachevskiu spaces, Uspekhi Mat. Nauk 42 (1987), no. 3(255), 13-38, 255 (Russian). MR896876 (88i:11066)

[12] Jian-Shu Li, Kloosterman-Selberg zeta functions on complex hyperbolic spaces, Amer. J. Math. 113 (1991), no. 4, 653-731, DOI 10.2307/2374843. MR.1118457 (92k:11055)

[13] G. D. Mostow, Strong rigidity of locally symmetric spaces, Annals of Mathematics Studies, No. 78, Princeton University Press, Princeton, N.J., 1973. MR0385004 (52 \#5874)

[14] S. Raghavan, On representation by hermitian forms, Acta Arith. 8 (1962/1963), 33-96. MR.0143734(26 \#1286)

[15] K. G. Ramanathan, Zeta functions of quadratic forms, Acta Arith. 7 (1961/1962), 39-69. MR0130863 (24 \#A717)

[16] John G. Ratcliffe and Steven T. Tschantz, On the representation of integers by the Lorentzian quadratic form, J. Funct. Anal. 150 (1997), no. 2, 498-525, DOI 10.1006/jfan.1997.3129. MR 1479550 (98h:11045)

[17] Carl Ludwig Siegel, On the theory of indefinite quadratic forms, Ann. of Math. (2) 45 (1944), 577-622. MR0010574 (6,38b)

[18] C. L. Siegel, Lectures on quadratic forms, Notes by K. G. Ramanathan. Tata Institute of Fundamental Research Lectures on Mathematics, No. 7, Tata Institute of Fundamental Research, Bombay, 1967. MR.0271028 (42 \#5911)

[19] Tonghai Yang, An explicit formula for local densities of quadratic forms, J. Number Theory 72 (1998), no. 2, 309-356, DOI 10.1006/jnth.1998.2258. MR.1651696 (99j:11034)

FaMAF-CiEM, Universidad Nacional de Córdoba, X5000HUA-Córdoba, Argentina

E-mail address: elauret@famaf.unc.edu.ar 